\title{
Reflets
}

Revue ontaroise d'intervention sociale et communautaire

\section{Les défis de l'intégration des nouveaux arrivants francophones en Ontario - Entrevue avec Antoine Dérose}

\section{Gertrude Mianda}

Volume 4, numéro 1, printemps 1998

Intervention en contextes minoritaires

URI : https://id.erudit.org/iderudit/026195ar

DOI : https://doi.org/10.7202/026195ar

Aller au sommaire du numéro

Éditeur(s)

Reflets : Revue ontaroise d'intervention sociale et communautaire

ISSN

1203-4576 (imprimé)

1712-8498 (numérique)

Découvrir la revue

Citer ce document

Mianda, G. (1998). Les défis de l'intégration des nouveaux arrivants

francophones en Ontario - Entrevue avec Antoine Dérose. Reflets, 4(1), 20-31.

https://doi.org/10.7202/026195ar

Tous droits réservés (C) Reflets : Revue ontaroise d'intervention sociale et communautaire, 1998
Ce document est protégé par la loi sur le droit d'auteur. L’utilisation des services d’Érudit (y compris la reproduction) est assujettie à sa politique d'utilisation que vous pouvez consulter en ligne.

https://apropos.erudit.org/fr/usagers/politique-dutilisation/ 


\section{Les défis de l'intégration des nouveaux arrivants francophones en Ontario}

L'entrevue qui suit a pour but de soulever les différentes situations problématiques vécues par les nouveaux arrivants à Toronto, et par la même occasion, en Ontario en général. Pour ce faire, nous avons préféré nous adresser à un homme de terrain, très actif et impliqué dans sa communauté et qui connaît particulièrement bien les enjeux de l'intégration des jeunes. Antoine Dérose est un travailleur social et occupe présentement le poste de conseiller de projet auprès du Conseil scolaire public du district Centre-Sud-Ouest de Toronto. Citoyen canadien d'origine haïtienne, il est père de trois enfants et réside à North York depuis 20 ans. Comme on pourra le constater, les conflits potentiels entre les générations sont au centre de beaucoup de difficultés rencontrées par les familles immigrantes et réfugiées. L'entrevue a été réalisée par Gertrude Mianda.

Reflets: Monsieur Dérose, vous travaillez avec les nouveaux immigrants, pouvezvous décrire en quoi consiste votre travail?

Antoine: Oui, je travaille avec les nouveaux immigrants, mais pas avec la population immigrante en général. Actuellement, je travaille beaucoup plus avec les élèves dans le système des écoles françaises de la communauté urbaine de Toronto. Mes clients sont pour la plupart des élèves qui ont des difficultés et qui ont besoin d'aide pour réussir dans leurs études. Ces élèves sont pour la plupart des ressortissants des pays africains francophones et des Caraibes. Mais mon expérience personnelle me permet de donner un peu d'information sur les problèmes auxquels font face beaucoup d'immigrants. 
En arrivant ici, ils doivent surmonter plusieurs obstacles et sont confrontés à de multiples barrières de divers ordres. Premièrement la barrière linguistique et deuxièment le choc culturel, c'est-àdire, la différence entre les valeurs sociales et morales des pays d'origine en comparaison avec celles du pays d'acceuil. Ce qui exige toujours une période d'adaptation.

Par ailleurs, je rencontre constamment dans mon travail un autre obstacle que les parents immigrants doivent franchir. Il s'agit du moyen utlisé dans l'application de la discipline. En effet, pour bien de parents et beaucoup d'élèves, la discipline corporelle a toujours servi comme méthode de maintien de l'autorité dans les rapports parents/enfants. La punition corporelle est donc perçue comme mesure de discipline. C'est quelque chose qui est normal dans les rapports parents/enfants. Pourtant ce genre de comportement est «criminalisé» ici à Toronto.

Voilà que ces parents qui ont été punis eux-mêmes corporellement et qui se sont toujours servis de ces punitions comme méthode de discipline, se trouvent confrontés à la loi au Canada. De son côté, l'enfant qui a toujours été puni, contrôlé par ses parents, se trouve dans une situation où on lui dit que personne n'a le droit de le toucher. La loi enlève aux parents un outil de discipline sans l'avoir remplacé par d'autres. Or, les parents sont désarmés face aux mauvais comportements de leurs enfants.

Quant à la barrière linguistique, les parents se retrouvent très souvent dans une position de dépendance vis-à-vis de leurs enfants, dans le sens où il est plus facile pour l'enfant d'apprendre une autre langue et de s'adapter à la nouvelle situation. L'enfant comprend rapidement la langue et la traduit pour ses parents. Il leur explique ce qui se passe dans la société. Il se produit en effet un renversement de situations dans les rapports parents/enfants. Cela est évidemment une conséquence de la barrière linguistique mais s'avère également un obsacle pour les parents qui voient une réduction de leur autorité dans une certaine mesure.

Il faut mentionner également comme obstacle, les stéréotypes et les préjugés auxquels doivent faire face les parents. Il arrive souvent qu'on juge les étrangers tout simplement par leur 
apparence. Si bien que certains immigrants, en dépit de leur très haut niveau d'instruction, ne sont embauchés que pour des travaux manuels ou des métiers qui ne correspondent pas à leur niveau de qualification. Par exemple, un médecin immigrant qui arrive ici, son titre ne lui est pas automatiquement reconnu. Il va falloir qu'il prouve qu'il est médecin, qu'il démontre tout ce dont il est capable.

Ce genre de discrimination touche également les minorités ethniques qui sont nées au Canada et dont les parents vivent au Canada depuis des générations. Il arrive souvent qu'on leur demande: "D'où viens-tu?». On les traite toujours comme des personnes d'origine africaine alors qu'ils sont canadiens comme «les vrais canadiens».

Reflets: Si je peux revenir à la question de comportement, vous avez parlé de méthodes corporelles utilisées par les parents immigrants et qui ne sont pas appliquées ici, vous avez surtout fait référence à l'enfant, de quel âge est cet enfant?

Antoine: Il s'agit des jeunes et des adolescents. En fait ici, dès la maternelle, dès l'école primaire, on commence à éduquer l'enfant à connaitre ses droits. Il est déjà protégé. Ce qui est une très bonne chose et même à encourager vivement. Cependant en contrepartie, les mécanismes de support pour harmoniser les relations parents/ enfants semblent faire défaut. Enfin, j'ai mentionné ceci tout simplement pour dire que j'ai vu des cas où des parents ont dû faire face à des charges criminelles parce qu'ils appliquaient la discipline qui est normalement acceptée dans leurs pays. Effectivement, il y a des parents qui perdent leurs enfants parce que les services de l'aide à l'enfance enlèvent l'enfant du foyer. Le grand problème est que les parents n'ont pas été avertis ou n'ont pas été préparés. On ne leur a pas donné des outils de remplacement pour qu'ils contôlent leurs enfants avec d'autres méthodes de discipline. Je ne veux pas insister sur ça, mais il fallait bien donner cet exemple. Dans le travail que je fais en tant que travailleur social, j'ai eu la chance de travailler avec beaucoup de groupes, et je vous avoue que c'est un problème que j'ai souvent rencontré. 
Reflets: D'après votre expérience, les institutions qui s'occupent de l'aide à l'enfance prêtent-elles oreille à ce que les parents avancent comme raison pour justifier leur méthode de discipline?

Antoine: En ce qui me concerne, j'aide les parents à développer d'autres méthodes de discipline. Par exemple, je leur demande d'accorder certains privilèges à l'enfant quitte à les lui ôter en guise de punition. De plus, je demande toujours aux parents et aux amis de ma communauté de développer des cours de parentage pour les jeunes. Mais malheureusement, le plus grand obstacle que je vois pour les immigrants de groupes ethniques venant des pays du Tiers-Monde, c'est une absence d'organisation communautaire, un manque d'accès aux services. Pourtant, il y a d'autres groupes comme par exemple les Juifs, les Italiens ou les Ukrainiens qui ont tout un système, tout un réseau ici sur place pour accueillir, encadrer et intégrer les nouveaux arrivants. Mais nous, plus spécialement les francophones du Tiers-Monde, n'avons pas ici de communauté pour faciliter notre intégration même sur le plan des relations parents/enfants.

Reflets: Y'a-t-il des raisons précises qui empêchent ces nouveaux venus originaires des pays du Tiers-Monde d'essayer de mettre en place des telles structures?

Antoine: Oui, il y a plusieurs raisons. Il y a le fait qu'ils sont triplement minoritaires. Ils le sont aussi bien par la langue et la culture que par le fait d'être des minorités ethniques. Tout cela rend les choses beaucoup plus difficiles. Un autre élément qui est tout aussi fatal pour certaines minorités ethniques est le manque de sens de communauté. J'ai essayé moi-même de monter quelque chose dans la communauté haïtienne avec l'aide des associations culturelles francophones. Actuellement encore, je travaille avec un groupe qu'on vient de mettre sur pied dans le but de répondre aux préoccupations et aux besoins des minorités francophones, entre autres, l'accès aux services et l'apprentissage de la langue. Pour ce faire, j'utilise un ami ou une connaissance qui va m'aider. En fin de compte, je déplore l'absence de services bien structurés, bien financés. Il faut préciser que je parle de la communauté haïtienne mais cela est aussi vrai pour toutes les autres communautés 
des minorités ethniques francophones avec lesquelles j'ai travaillé à Toronto.

Reflets: Peut-on en déduire qu'il y a un effort à faire de part et d'autre, autant du côté gouvernemental que de celui des communautés ethniques?

Antoine: Définitivement. Cependant, je suis pour la responsabilité individuelle. Je ne crois pas à la dépendance. Je ne pense pas qu'on rendrait service à quelqu'un en mettant tout à sa disposition. Ce serait lui rendre un mauvais service. Il faut un effort de la part de l'individu; il en va de même pour la communauté. J'admets que les communautés ont besoin des services du gouvernement. Mais nous devons travailler en partenariat avec celui-ci ou d'autres agences qui existent et qui peuvent fournir des services.

Reflets: Puisque vous évoquez les structures pouvant faciliter l'intégration des immigrants, pouvez-vous m'expliquer relativement comment s'opère l'intégration des personnes immigrantes?

Antoine: D'après mon expérience personnelle, l'intégration ne se fait pas rapidement. Dans certains cas, l'intégration ne se fait pas du tout. Il faut attendre la deuxième génération, c'est-à-dire des enfants qui ont été éduqués et élevés au Canada. En effet, les premiers arrivés d'une culture maintiennent une relation presque morbide avec la culture d'origine, à tel point que la culture d'origine évolue et les laisse sur place. Je connais un cas où dans la communauté italienne, des Italiens, qui sont venus après la guerre, ont gardé en mémoire la vie qu'ils ont laissée en Sicile. Quarante ans après, ils sont rentrés chez eux et ils ont été très choqués par les changements qui sont intervenus là-bas, car eux, ils ont réussi à maintenir la vie culturelle qu'ils avaient reçue de leurs parents. Après avoir travaillé très dur pour aller prendre leur retraite chez eux, ils ont trouvé que chez eux, tout avait changé. Ils voyaient des photos de femmes et d'hommes nus affichées aux murs, des jeunes garçons et des jeunes filles qui s'embrassaient partout, tout avait changé... Alors, ça arrive souvent quand on a "romantisé» et gardé précieusement les valeurs de sa société et ceci est aussi une barrière à l'intégration. 
Sur le plan économique, l'intégration est de plus en plus difficile. Les choses ont beaucoup changé. Il y a vingt ans, on pouvait entrer au Canada sans éducation, sans savoir lire ni écrire. On pouvait se trouver du travail. En travaillant très durement, on parvenait même à s'acheter une belle maison, une belle voiture, etc. Actuellement, ce n'est plus le cas. Le marché d'emploi est comme saturé, même pour les Canadiens d'origine. Maintenant, il faut au moins un diplôme d'études secondaires. C'est la raison pour laquelle je travaille avec les élèves pour les pousser, pour leur faire comprendre que s'ils n'ont pas une bonne instruction, avec toutes les coupures qui se font dans les services sociaux, ils ne peuvent plus compter sur le chèque d'aide sociale.

Le marché du travail devient de plus en plus un marché de la haute technologie. Il exige des connaissances de plus en plus élevées au point qu'on ne peut plus se contenter de peu de connaissances. Des amis haitiens m'ont dit que quand ils sont entrés à Montréal en 1972, on cherchait des gens pour travailler. Le marché de l'emploi était encore très ouvert. Les gens pouvaient travailler dans des manufactures. Actuellement, la plupart sont fermées et n'existent plus.

L'intégration devient plus difficile encore parce qu'il faut apprendre non seulement la langue, mais également se recyler, notamment dans l'usage de la technologie, comme par exemple savoir se servir de l'ordinateur.Tous les stigmates qui sont attachés aux services sociaux rendent les choses encore plus difficiles. D'un autre côté, il faut faire face à ce gouvernement qui s'acharne à blâmer, à couper tous les services fournis aux plus démunis et qui ne facilite pas l'intégration des nouveaux immigrants.

Reflets: En relevant les difficultés d'intégration des personnes immigrantes, trouvezvous d'après votre expérience qu'elles rencontrent les mêmes problèmes, indépendamment de leur sexe?

Antoine: Non, je pense qu'il y a une dimension universelle aux problèmes des femmes. Cela ne concerne pas seulement les femmes immigrantes, mais les femmes en général. Dans un pays comme le Canada, la femme immigrante, à l'instar de toutes les autres femmes, 
doit se trouver du travail. La femme ne reste plus à la maison avec les enfants comme cela se passait avant. Cependant, la femme immigrante est confrontée aux problèmes de stéréotypes, de préjugés, de la barrière linguistique, de manque de soutien et de support. La situation de la femme immigrante devient encore beaucoup plus problématique dès qu'elle est par exemple monoparentale. Dans ces circonstances, elle est la seule à supporter ses enfants et parfois ses propres parents. Le soutien aux parents constitue malheureusement encore une priorité pour la femme comparativement aux hommes qui peuvent s'en défaire.

Reflets: Comme vous l'avez souligné, les immigrants doivent surmonter certaines barrières en vue de leur intégration. D'après votre expérience quelles sont leurs stratégies pour réussir leur intégration?

Antoine: Je pense que pour l'intégration de nouveaux immigrants, il faudrait un support, un soutien communautaire couplé à un plan de financement des services du gouvernement. Il s'agit des services tels que l'éducation, les soins de santé, l'information.

Les gens utilisent leurs connaissances personelles, leurs ressources, leurs amis au lieu d'un système intégré, un système administré par le gouvernement. Je reçois dans bien des cas des appels de gens et je m'occupe personnellement de la personne ou je la recommande à un agent. Car les communautés francophones ont des ressources bien limitées et je pense que c'est important que leur communauté d'origine les aident à s'intégrer. Les communautés ethno-culturelles doivent donc s'organiser elles-mêmes pour offrir ne serait-ce que les services minimum. En fait, l'idéal serait de parvenir à une connexion entre les services d'immigration et les services communautaires afin de guider les nouveaux arrivants dès l'aéroport. Organiser pour eux, par exemple, des listes de contacts et de ressources disponibles, entre autres, au niveau des communautés.

Reflets: Faut-il donc faire du recrutement au niveau des communautés?

Antoine: Absolument, cela nécessite l'intervention du gouvernement. Il faudrait qu'il y ait des gens payés par le gouvernement pour un 
service de base, un service d'accueil, un service de renseignements, un service d'information, d'accompagnement et de traduction. Il n'y a pas un service de base pour répondre à ces besoins.

Reflets: Vous venez de faire allusion tantôt au soutien de la commuauté. Qu'entendez-vous par là? S'agit-il de la communauté d'origine des immigrants?

Antoine: Oui,je pense que leur communauté d'origine devrait également offrir ce soutien car le nouvel arrivé se sent toujours beaucoup plus à l'aise quand il a affaire avec des gens de sa propre culture. Je me souviens quand je venais d'arriver ici à Toronto,j'ai cherché à rencontrer des Haïtiens. C'était tellement important pour moi de savoir qu'il y avait ici quelqu'un de mon pays, de ma culture, qui connaissait ma langue et avec qui je pouvais entrer en contact! J'ai pu rencontrer quelques Haitiens dans le cercle francophone, qui parlaient créole. C'est important car la langue d'origine unit.

Reflets: Si je vous comprends bien, vous pensez qu'il est relativement facile pour une personne francophone qui arrive à Toronto de s'intégrer en passant par sa communauté d'origine?

Antoine: Cela facilite énormément. Je crois qu'il faut contacter les cercles francophones pour certaines choses, mais ils sont extrêmement limités. Et en plus, ils ne touchent que ceux qui parlent français. Si vous parlez lingala ou swahili ou une autre langue, il vous faudra d'abord apprendre soit le français ou l'anglais. C'est pourquoi il faudrait des services dans certaines langues d'origine qui sont acceptées au niveau international. D'autre part, je pense que ce serait important que les Canadiens connaissent plusieurs langues. En effet, quand je parle de service, je vois d'abord des personnes moins nanties. Je ne vise pas nécessairement celles qui sont instruites. Ces personnes peuvent se débrouiller seules, notamment pour contacter un avocat ou un médecin. 
Reflets: Revenons si vous le permettez à la problématique d'intégration des jeunes. Voyez-vous, d'après votre expérience, une différence dans l'intégration des jeunes comparativement à celle des adultes?

Antoine: L'intégration des jeunes est plus rapide mais peut être un peu plus brutale car il y a trop de pression sur eux. Dans le milieu scolaire, la pression du groupe est beaucoup plus forte. Les parents demeurent isolés chez eux et ne subissent pas la même pression. Cette disparité de l'intégration entraîne des conflits entre les générations.

Reflets: Pouvez-vous illustrer cela par quelques exemples?

Antoine: Sur le plan économique, il n'y a pas vraiment de conflit parce que les jeunes restent toujours dépendants des parents, même dans les cas où ces derniers ne travaillent pas. Les jeunes ne recevant pas directmement l'assistance sociale, la dépendance financière aux parents demeure. Mais l'intégration des jeunes se fait plus rapidement sur les plans culturel et linguistique. Ils apprennent plus vite la langue. Ils s'habillent et se comportent comme ceux qu'ils ont trouvés sur place. Le besoin de s'assimiler au groupe est beaucoup plus accentué. Prenons l'exemple des pays islamiques où certains contacts entre les jeunes de sexe différent ne sont pas bien vus. Il est plus difficile pour ces parents d'envoyer leurs enfants au cinéma, de laisser sortir les filles ou les garçons ensemble. À ce niveau, les jeunes s' intégrent rapidement et n'y trouvent aucun mal pendant que les parents n'acceptent pas encore ce genre de fréquentations entre les jeunes garçons et les jeunes filles. Le fait que les jeunes demandent à leurs parents de rejeter toutes les valeurs culturelles et religieuses en plus du renversement de rôle au niveau linguistique sépare les parents de leurs enfants.

Reflets: Comment réagissent les parents face à ce conflit culturel entre les générations compte tenu du sexe des enfants?

Antoine: En général, les parents veulent plus protéger les filles dans certaines cultures. C'est là encore une autre source de conflit car le jeune frère peut se permettre bien des choses qui sont interdites à la fille. On a même vu dans certains cas la mère avoir beaucoup plus de déférence envers son fils. 
Je suis favorable à l'intégration plutôt qu'à l'assimilation; car l'assimilation est un rejet total de la culture d'origine, un vrai processus d'acculturation qui se fait au profit de la culture d'adoption. Et quand il n'y a pas de barrière à la maison, les jeunes rejettent tout plus vite. Cependant, un jeune musulman ne peut pas rejeter les valeurs qui lui conferent une certaine autorité, un certain respect, un certain droit. Mais en fin de compte, les jeunes sélectionnent les valeurs. Il y a encore des jeunes qui portent le voile.

Cependant, cette intégration est certes facile mais elle n'est pas totale. Il y a certaines valeurs linguistiques et culturelles que les jeunes ne peuvent abandonner. Souvent ils parlent la langue et suivent les règles, les coutumes du pays d'accueil mais ils gardent leurs valeurs religieuses.

Reflets: Mais qu'en est-il des jeunes filles?

Antoine: Il y a un point qui est intéressant, c'est que les garçons ont une certaine arrogance. Ils ont certains avantages que leurs soeurs, leurs amies, leurs cousines n'ont pas. Ils peuvent sortir comme ils veulent. Ceci explique le fait que le conflit survient plus fréquemment et de manière presque permanente entre les jeunes filles et les parents. Heureusement que ces jeunes filles peuvent travailler, passer leur examen de conduite, s'acheter une voiture, bien que ces choses là ne soient pas encouragées par la culture et les valeurs religieuses. Les parents voudraient également voir leurs filles devenir médecins ou avocates. Mais je n'oserais pas vraiment me prononcer davantage sur ce sujet parce que je ne l'ai pas étudié en profondeur. Je n'aimerais pas tomber dans le piège de créer des stéréotypes.

Reflets: Nous n'avons fait mention jusqu'ici que des personnes immigrantes. $Y$ a-t-il une différence entre elles et les réfugiés au Canada?

Antoine: Oui, il y a une grande différence en ce sens que la personne qui arrive comme immigrante a directement accès à toute une série de services auxquels celui qui arrive comme réfugié n'a pas accès automatiquement. On se rend compte de plus en plus que s'il n'y 
a pas une structure familiale ou communautaire pour accueillir celui qui fait la demande d'asile, il lui est très difficile d'aller à l'école ou de recevoir de l'aide sociale. Il y a même des cas où on le retient en détention.

Il y a vraiment une grande différence entre les besoins des réfugiés et ceux des immigrants. Les ressources personelles et individuelles de l'immigrant font toute la différence. Il suffit qu'il ait du support pour s'en tirer plus facilement. En fin de compte, il ne lui restera qu'à surmonter l'obstacle linguistique et le choc culturel.

Pour les réfugiés, le problème est plus complexe.J'ai été appelé plusieurs fois pour servir d'interprète parce qu'il y a tellement de réfugiés francophones. Il leur faut également des ateliers d'information sur les possibilités qui existent sur le marché du travail, l'accès aux services, le comportement dans certains milieux. Bref, il faut un partenariat entre les communautés et le gouvernement.

Reflets: Comment comparez-vous la situation des immigrants et des réfugiés au niveau des jeunes?

Antoine: C'est beaucoup plus facile pour les jeunes immigrants que pour les jeunes réfugiés. Dans le temps, lorsque la demande d'asile avait été faite, on avait automatiquement accès à plusieurs services. Mais il y a toute une série de changements qui sont inervenus qui privent les demandeurs d'asile de tous ces services. Par exemple, les jeunes réfugiés ne peuvent pas avoir accès au prêt-bourse et comme ils n'ont pas non plus le droit de travailler, ils ne peuvent pas étudier. Ils n'ont même pas droit à la carte d'assurance sociale. C'est là une façon de décourager les demandeurs d'asile. La situation des réfugiés devrait inciter les communautés culturelles à se battre davantage.

Reflets: Quelles sont en fin de compte, selon vous, les dispositions que l'on peut prendre pour améliorer ou faciliter l'intégration de nouveaux immigrants?

Antoine: Il faut un financement conséquent. Il faut également permettre l'accès aux services. Le gouvernement devrait travailler en partenariat avec les communautés. Il faut que les immigrants soient préparés à devenir des citoyens canadiens. Les coupures dans les 
services offerts aux immigrants, aux refugiés ainsi qu' aux adultes sont regrettables. Elles portent un coup fatal à l'organisation de ces services.

Nous vivons dans un milieu anglophone, il faut donc apprendre l'anglais. Les services d'accueil devraient comprendre une composante qui enseigne l'anglais comme langue vivante. C'est un service primordial pour l'intégration parce que si tu ne parles pas l'anglais, tu seras condamné à être demandeur d'aide sociale toute ta vie. Le gouvernement ne finance plus les projets de groupes.

Reflets: Et si les communautés s'organisent, peuvent-elles avoir le financement du gouvernement?

Antoine: Oui, les communautés juives, ukrainiennes et italiennes reçoivent énormément l'aide du gouvernement. C'est une question d'organisation car le gouvernement doit justifier pourquoi il donne aux uns et pas aux autres comme les francophones qui ne répondent pas encore aux critères arrêtés par le gouvernement pour mériter ce financement. Il faut donc avoir une structure sur place qui ne dépende pas d'une seule personne ou d'un petit groupe mais qui a un caractère d'institution durable.

Reflets: Nous sommes arrivés au terme de cette entretien, Monsieur Dérose, et je vous remercie beaucoup pour votre disponibilité.

Antoine: C'est moi qui vous remercie, madame. 